\title{
CONTROL OF WEEDS IN TOBACCO SEEDBEDS
}

Among the chemicals used last year for the control of weeds in tobacco seedbeds Weedrench (allyl alcohol) proved the best. Natrin and Premerge, at the concentrations used, were very effective in controlling weeds, but they considerably reduced germination of the tobacco seed. Weedrench not only controlled weeds remarkably well, but the number of seedlings per plot was significantly higher than in the control and otherwise-treated plots. It was also observed that seedlings growing in Weedrench-treated beds developed faster and were of a greener color. Apparently, this chemical controlled some kind of soil-borne organism or interfered with some soil property which resulted in a better stand and growth of seedlings. According to our results, Weedrench can be used as a drench at the rate of $11 / 2$ gallons in 100 gallons of water per 1,000 square feet of seedbed. The tobacco seed can be planted a week after the material is applied.

Héctor R. Cibes

Plant Physiology Deparlment

Alberto Amy

Seed Farms Division 